\title{
Valoración de la herramienta Google Drive durante el trabajo colaborativo universitario
}

\section{Assessment of the Google Drive tool during university collaborative work}

Avaliação da ferramenta Google Drive durante o trabalho colaborativo da universidade

\author{
Miguel Ángel Rangel Romero \\ Universidad de Guadalajara, México \\ marangel@cusur.udg.mx \\ https://orcid.org/0000-0002-1717-1063 \\ Adriana Lorena Îniguez Carrillo \\ Universidad de Guadalajara, México \\ adriana.carrillo@cusur.udg.mx \\ https://orcid.org/0000-0001-9753-716X \\ Abraham Jair López Villalvazo \\ Universidad de Guadalajara, México \\ abraham@cusur.udg.mx \\ https://orcid.org/0000-0002-8877-9703
}




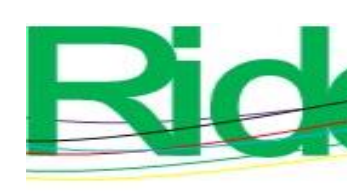

Revista Iberoamericana para la
Investigación y el Desarrollo Educativo
ISSN $2007-7467$

\section{Resumen}

El objetivo de esta investigación, cuyo enfoque fue descriptivo y transversal, se enfocó en conocer la opinión de estudiantes universitarios sobre su experiencia al utilizar Google Drive para trabajar de manera colaborativa. El tema abordado con dicha estrategia colaborativa fue la elaboración del planteamiento del problema de una investigación, apartado constituido por la contextualización del tema, su justificación y las preguntas y objetivos de investigación. Los participantes fueron alumnos inscritos en las asignaturas Metodología y Práctica de la Investigación de la licenciatura en Negocios Internacionales (LINI) y Metodología de la Investigación de la carrera de Médico Cirujano Partero (MCP) del ciclo escolar 19-B (antes de la pandemia covid-19) en el Centro Universitario del Sur (CUSur). Los resultados demuestran que Google Drive resultó de gran ayuda para desarrollar cada una de las tareas indicadas. De hecho, los alumnos identificaron como atractivo su uso, pues al poder compartir y modificar documentos en la nube pudieron vencer las barreras de los horarios y de la presencia física.

Palabras clave: enseñanza-aprendizaje, herramientas digitales, trabajo colaborativo.

\section{Abstract}

Collaborative work is recognized as a teaching-learning strategy in which in a school environment it is identified by working in small or few teams with regard to the group in general. Among the main essential characteristics, it can be identified that each member must understand the objective clearly and efficiently through activities generated by a “community". In this study, the use of Google Drive will be shown, where students were able to build and edit Word documents from the place where they were without the requirement to be in the same room. With the objective of knowing the opinion about their experience when using Google Drive collaboratively, this descriptive-transectional research had a sample that was made up of 65 students from three groups of the "Research Methodology and Practice" course from the degree of International Business and "Research Methodology" course of the degree of Surgeon and Obstetrician of the University Center of the South of the University of Guadalajara. A digital questionnaire was applied during November and December of 2019, collecting the information to describe their appreciations and experiences. Taking as results that the majority of students $(92 \%)$ consider that Google Drive collaborated to achieve the learning objective and $97 \%$ of students have been able to work 


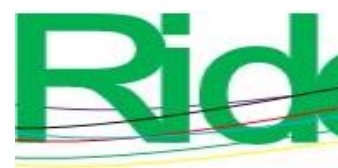

Revista Iberoamericana para la
Investigación y el Desarrollo Educativo
ISSN $2007-7467$

collaboratively using Google Drive. Among the main conclusions, it was noted that the free Google Drive offered advantages of synchronous collaboration among team members since communication among the majority of students improved during the assigned activities and became pragmatic for the fulfillment of school activities.

Keywords: teaching-learning, digital tols, collaborative work.

\section{Resumo}

O objetivo desta pesquisa, cuja abordagem foi descritiva e transversal, centrou-se em conhecer a opinião de estudantes universitários sobre a sua experiência ao utilizar o Google Drive para trabalhar de forma colaborativa. $\mathrm{O}$ tema abordado com esta estratégia colaborativa foi a elaboração de um enunciado do problema de pesquisa, uma seção composta pela contextualização do tema, sua justificativa e as questões e objetivos da pesquisa. Os participantes foram alunos matriculados nas disciplinas de Metodologia de Pesquisa e Prática do Curso de Negócios Internacionais (LINI) e Metodologia de Pesquisa do Cirurgião de Obstetrícia (MCP) da carreira do 19-B ano letivo (antes da pandemia de cobiça). -19) no Centro Universitario del Sur (CUSur). Os resultados mostram que o Google Drive foi de grande ajuda no desenvolvimento de cada uma das tarefas indicadas. De facto, os alunos identificaram a sua utilização como atractiva, uma vez que ao poder partilhar e modificar documentos na nuvem, conseguiram ultrapassar as barreiras dos horários e da presença física. Palavras-chave: ensino-aprendizagem, ferramentas digitais, trabalho colaborativo.

Fecha Recepción: Octubre 2020

Fecha Aceptación: Marzo 2021

\section{Introducción}

El trabajo colaborativo se caracteriza porque los productos logrados son enriquecidos por la aportación de todos los miembros de un equipo, lo que de manera paralela contribuye a fortalecer el aprendizaje de cada individuo. Maldonado (2007) lo concibe del siguiente modo: 

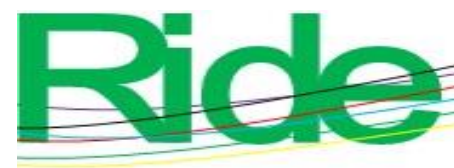

Revista Iberoamericana para la Investigación y el Desarrollo Educativo ISSN $2007-7467$

El trabajo colaborativo, en un contexto educativo, constituye un modelo de aprendizaje interactivo, que invita a los estudiantes a construir juntos, lo cual demanda conjugar esfuerzos, talentos y competencias, mediante una serie de transacciones que les permitan lograr las metas establecidas consensuadamente. Más que una técnica, el trabajo colaborativo es considerado una filosofía de interacción y una forma personal de trabajo, que implica el manejo de aspectos, tales como el respeto a las contribuciones individuales de los miembros del grupo (p. 268).

En esta estrategia didáctica, el acompañamiento en la conformación de los equipos es una tarea clave para el asesor o guía, pues al interactuar en las sesiones de trabajo se podrán promover las responsabilidades de cada miembro del equipo, lo que fomentará valores como la solidaridad y la cooperación, así como el pensamiento crítico y el respeto por las opiniones ajenas (Ríos, Chamba, Zumba y Pardo, 2019). En palabras de Romero et al. (2019), "este modelo también induce a la motivación de los estudiantes por situaciones del mundo real, porque se pueden desarrollar entornos muy reales, de tal manera que las personas trabajan de esa forma" (p. 57). Para Thomas (2014) se debe prever que los equipos conformados no superen los 6 integrantes para que se pueda interactuar debidamente y para que los grupos no se tornen difíciles de manejar. 
Tabla 1. Conceptualización de trabajo colaborativo

\begin{tabular}{|l|l|}
\hline Autores & Conceptualizaciones \\
\hline Guitert y Giménez & $\begin{array}{l}\text { Proceso en el que cada individuo aprende más de lo que } \\
\text { aprendería por sí solo, fruto de la interacción de los integrantes } \\
\text { del equipo. El trabajo colaborativo se da cuando existe una } \\
\text { reciprocidad entre un conjunto de individuos que saben } \\
\text { diferenciar y contrastar sus puntos de vista de tal manera que } \\
\text { llegan a generar un proceso de construcción de conocimiento. }\end{array}$ \\
\hline Panitz y Panitz (1998) & $\begin{array}{l}\text { Proceso de interacción cuya premisa básica es la construcción } \\
\text { de consenso. Se comparte la autoridad y entre todos se acepta } \\
\text { la responsabilidad de las acciones del grupo. }\end{array}$ \\
\hline Gros (2000) & $\begin{array}{l}\text { Poceso en el que las partes se comprometen a aprender algo. } \\
\text { del grupo es realizado en colaboración. Es el grupo el que } \\
\text { decide cómo realizar la tarea, qué procedimientos adoptar, y } \\
\text { cómo dividir el trabajo o tareas a realizar. La comunicación y } \\
\text { la negociación son claves en este proceso. }\end{array}$ \\
\hline
\end{tabular}

Fuente: Revelo, Collazos y Jiménez (2017)

Para el proceso de enseñanza-aprendizaje las tecnologías de la información y comunicación han posibilitado un abanico de herramientas que permiten el trabajo colaborativo de manera presencial o a distancia. Por ejemplo, el uso de chats (a través de Whatsapp, Facebook, etc.) facilita la toma de decisiones y la comunicación permanente, rápida, eficaz y en tiempo real. Asimismo, los servicios de videollamadas permiten el desarrollo de reuniones entre dos o más interesados e incluso la divulgación en pantalla de documentos y diapositivas. Además, los blogs permiten comparar comunicaciones, avances, compilación de datos o contenidos sobre determinado tema.

En este contexto de múltiples oportunidades digitales, Google se caracteriza por ser una empresa especializada en productos y servicios electrónicos, informáticos y vinculados mediante internet que facilitan la divulgación de información y la comunicación. Para Aruquipa, Chávez y Reyes (2016) "Google es una estrategia que enriquece y fortalece el proceso de aprendizaje en los estudiantes" (p. 26). Sus principales plataformas son Chrome para equipos computacionales de escritorio y laptops, y Android para dispositivos móviles (celulares y tabletas). Entre los diversos recursos que ofrece esta empresa se puede mencionar 


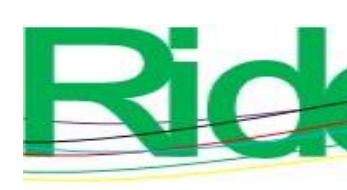

Revista Iberoamericana para la
Investigación y el Desarrollo Educativo
ISSN $2007-7467$

Google Drive, servicio que fue usado en el presente estudio porque permite almacenar, compartir y editar documentos de forma grupal. En este caso concreto, los participantes fueron los estudiantes de las materias Metodología y Práctica de la Investigación de la licenciatura en Negocios Internacionales (LINI) y Metodología de la Investigación de la carrera de Médico Cirujano Partero (MCP) del Centro Universitario del Sur (CUSur).

El CUSur es una institución de la Red de la Universidad de Guadalajara (UdeG), y cuenta con un total de 8623 alumnos distribuidos en los 19 programas de licenciatura y en los 9 de posgrado que se ofertan en dicha casa de estudios. Este centro está ubicado en Ciudad Guzmán, cabecera municipal de Zapotlán El Grande, Jalisco (México), población que cuenta con 105423 habitantes (Instituto de Información Estadística y Geográfica de Jalisco [IIEG], 2019). Está considerada como la segunda ciudad del estado por su factibilidad como polo de desarrollo industrial. Se localiza a 125 kilómetros de Guadalajara (segunda ciudad en importancia del país), a 40 minutos de la Ciudad de Colima (capital del estado con el mismo nombre) y a 90 minutos de Manzanillo (puerto de entrada y salida de los mercados de la cuenca del Pacífico).

Por otra parte, los propósitos de las asignaturas que cursaban los alumnos que participaron en el presente estudio son los siguientes:

- Investigar y analizar las metodologías que de manera predominante se han aplicado en la generación y aplicación del conocimiento científico en su campo profesional.

- Localizar y analizar literatura especializada en torno al área de investigación propuesta.

- Plantear la problemática de un área profesional con los antecedentes científicos que la sustentan.

- Aplicar el método y diseño de investigación más adecuado para el área de indagación profesional.

Para el abordaje de los temas y subtemas se ha elaborado el diseño instruccional como estrategia de enseñanza-aprendizaje, siguiendo el modelo ADDIE (acrónimo de los términos análisis, diseño, desarrollo, implementación y evaluación), el cual es un esquema teórico que sirve para analizar y elaborar con detenimiento cada apartado de actividades de formación o diseño instruccional. 


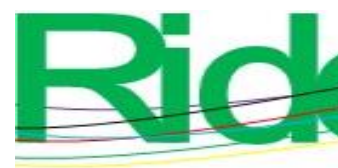

Revista Iberoamericana para la
Investigación y el Desarrollo Educativo
ISSN $2007-7467$

cooperación entre pares, el autoaprendizaje y las habilidades de comunicación. Al respecto, Mora-Vicarioli y Hooper-Simpson (2016) recomiendan que en estos casos se empleen herramientas como el foro o el chat grupal para promover la negociación entre los grupos de trabajo.

\section{Objetivo de la investigación}

El trabajo colaborativo ha encontrado en internet un marco ideal para facilitar sus actividades y el logro de sus propósitos. Al respecto, Esteve (citado por Barrios y Casadei, 2014) afirma que estas herramientas ayudan al desarrollo de destrezas y, sobre todo, de actitudes basadas en una alfabetización tecnológica, crítica, colaborativa y creativa. Por tal motivo, en esta investigación, a partir del diseño instruccional, de forma colaborativa se pretende lo siguiente:

- Conocer la opinión de estudiantes universitarios sobre su experiencia al utilizar Google Drive para trabajar de manera colaborativa.

El tema abordado con dicha estrategia colaborativa fue la elaboración del planteamiento del problema de una investigación, apartado constituido por la contextualización del tema, su justificación y las preguntas y objetivos de investigación. En tal sentido, la pregunta de investigación formulada fue la siguiente: ¿el uso de Google Drive facilita la creación y uso de documentos compartidos entre alumnos universitarios en un ambiente de trabajo colaborativo?

\section{Participantes}

La muestra estuvo constituida por los 71 alumnos inscritos en las asignaturas Metodología y Práctica de la Investigación de la licenciatura en Negocios Internacionales (LINI) y Metodología de la Investigación de la carrera de Médico Cirujano Partero (MCP) del ciclo escolar 19-B (antes de la pandemia covid-19) en el Centro Universitario del Sur (CUSur). Se tomaron estos grupos por conveniencia del investigador, y de los 71 cuestionarios se pudieron validar 65 . 


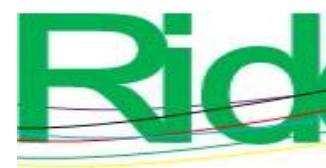

Revista Iberoamericana para la Investigación y el Desarrollo Educativo ISSN 2007 - 7467

\section{Metodología}

El estudio tuvo un enfoque descriptivo y transversal, su "procedimiento consiste en ubicar en una o diversas variables a un grupo de personas u otros seres vivos, objetos, situaciones, contextos, fenómenos, comunidades, etc., y proporcionar su descripción” (Hernández, R., Fernández, C. y Baptista, P., 2014, p. 155). Y en lo particular se conformaron equipos de cuatro o cinco integrantes, cada uno de los cuales creó un grupo de WhatsApp, así como una carpeta compartida en Google Drive. Cada integrante tenía permisos de administrador para editar los documentos que se trabajarían.

Con el fin de conocer la opinión de los alumnos en torno a la actividad desarrollada, se elaboró un cuestionario digital mediante la herramienta Formularios Google, para lo cual se tomaron en cuenta las investigaciones de Brescó y Verdú (2014) y Castellanos y Martínez (2013). El instrumento final quedó conformado por 16 reactivos redactados de manera clara y sencilla, y fue respondido de manera anónima y voluntaria.

\section{Resultados}

Como se mencionó, de los cuestionarios aplicados 65 resultaron válidos. A continuación, se presentan los principales resultados. De forma general, se puede indicar que los participantes fueron 51 mujeres y 14 hombres (figura 2).

Figura 2. Género de los participantes

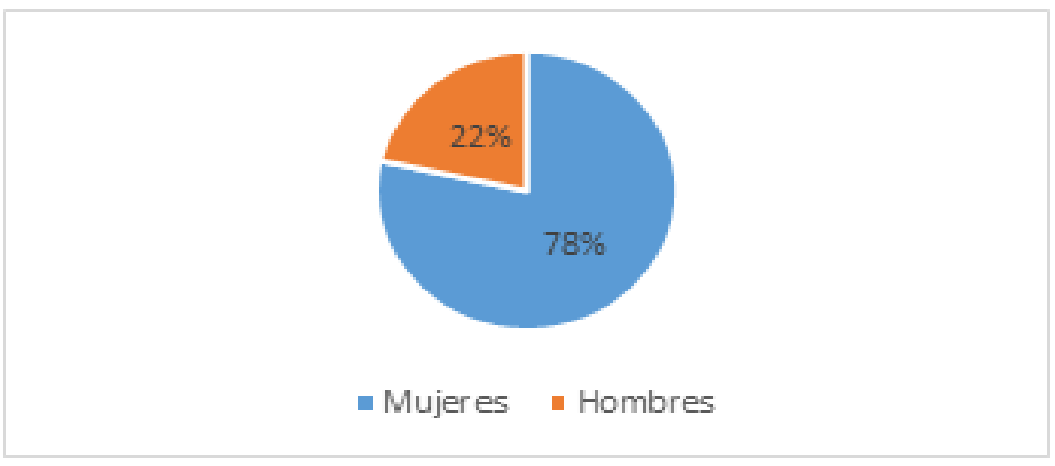

Fuente: Elaboración propia

En cuanto al conocimiento de Google Drive, solo 10 participantes indicaron que desconocían la plataforma, mientras que el resto sí había trabajado en ella. 

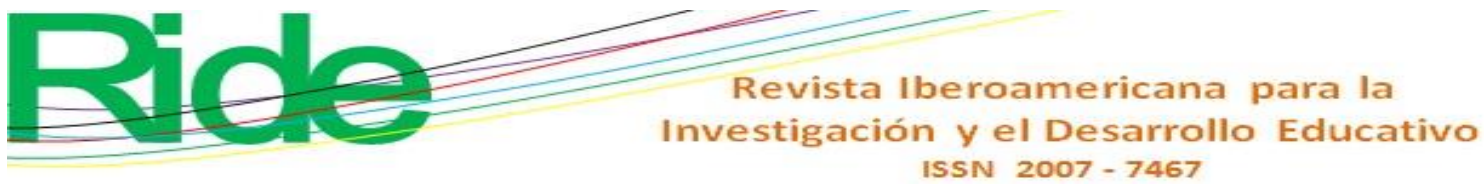

Figura 5. ¿Te resultó fácil aportar y modificar documentos de Google Drive?

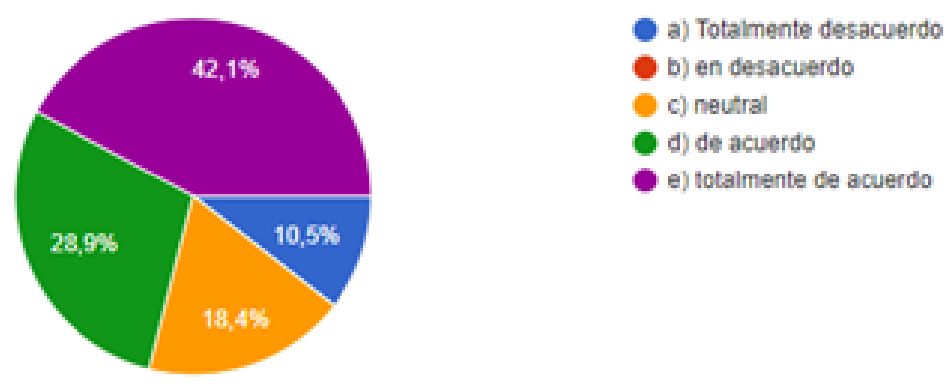

Fuente: Elaboración propia

Además, se puede indicar que a $15.8 \%$ de los participantes Google Drive no les facilitó la realización de las actividades.

Figura 6. ¿Con Google Drive se te facilitó hacer las actividades indicadas?

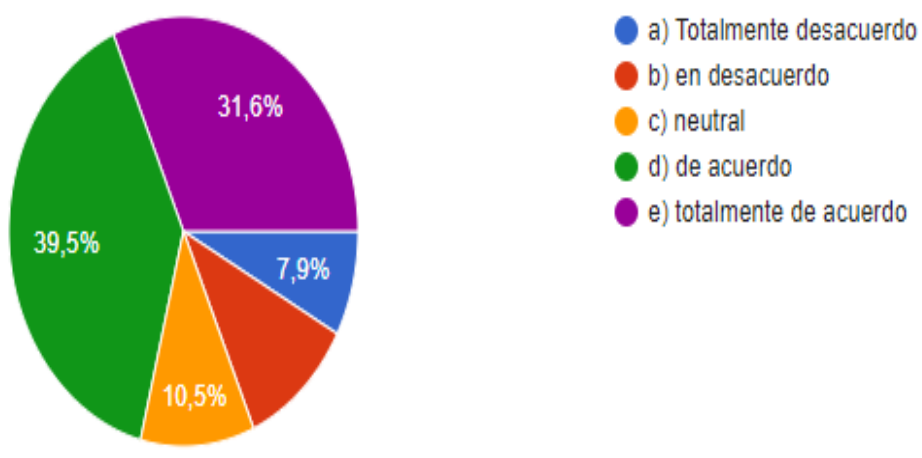

Fuente: Elaboración propia

Por otra parte, la mayoría de los alumnos (92.1\%) considera que Google Drive colaboró para el logro del objetivo de aprendizaje. 

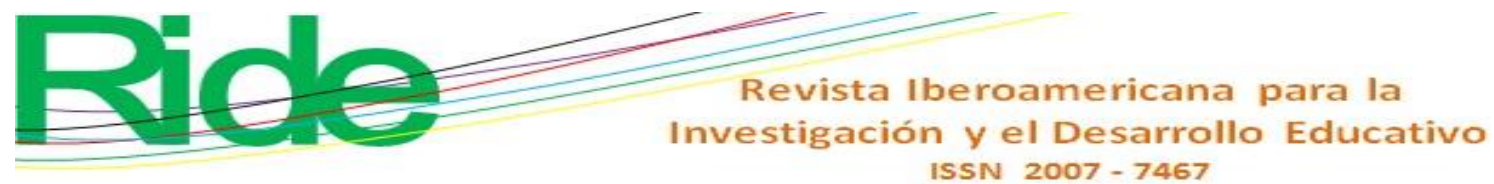

Figura 7. ¿Consideras que Google Drive colaboró para el logro del objetivo de aprendizaje?

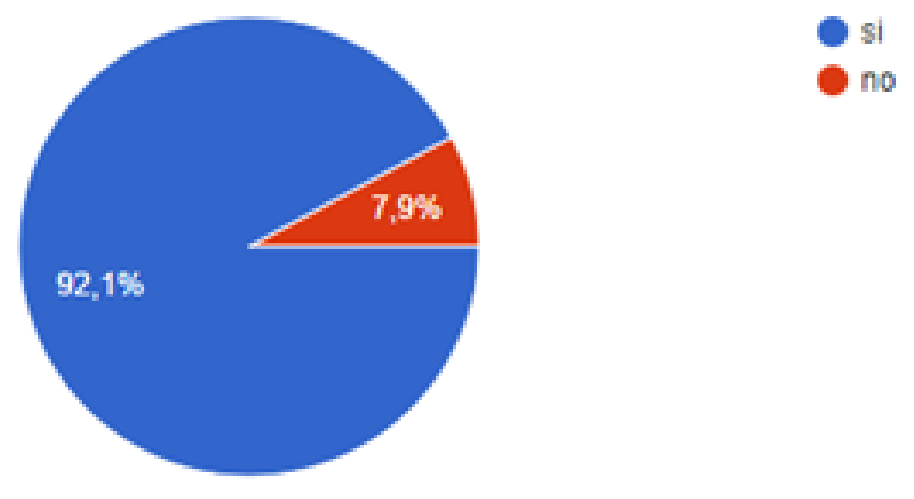

Fuente: Elaboración propia

Asimismo, casi $90 \%$ de los alumnos consideró que Google Drive ayudó a realizar los trabajos en grupo.

Figura 8. ¿Piensas que el uso de Google Drive te ayudó a realizar trabajos en grupo?

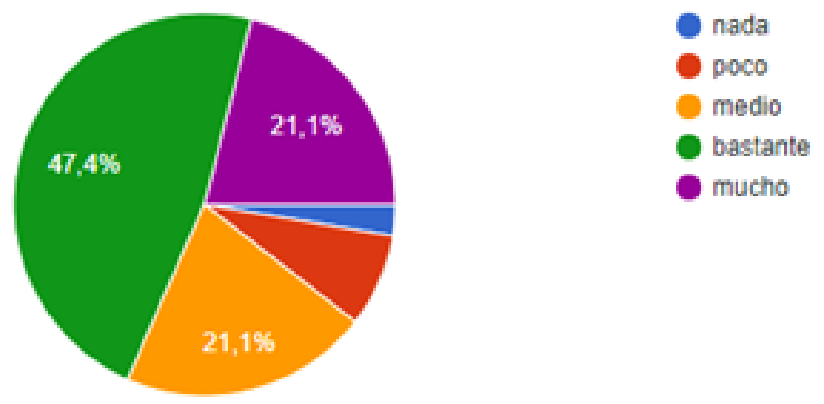

Fuente: Elaboración propia

También se pudo constatar que 63 alumnos han podido trabajar de manera colaborativa usando Google Drive, mientras que solo dos opinaron lo contario. 


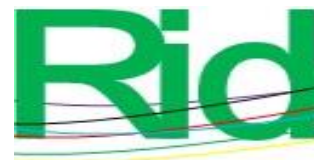

Revista Iberoamericana para la
Investigación y el Desarrollo Educativo
ISSN $2007-7467$

Figura 9. ¿Te ha resultado sencillo trabajar de manera colaborativa en Google Drive?
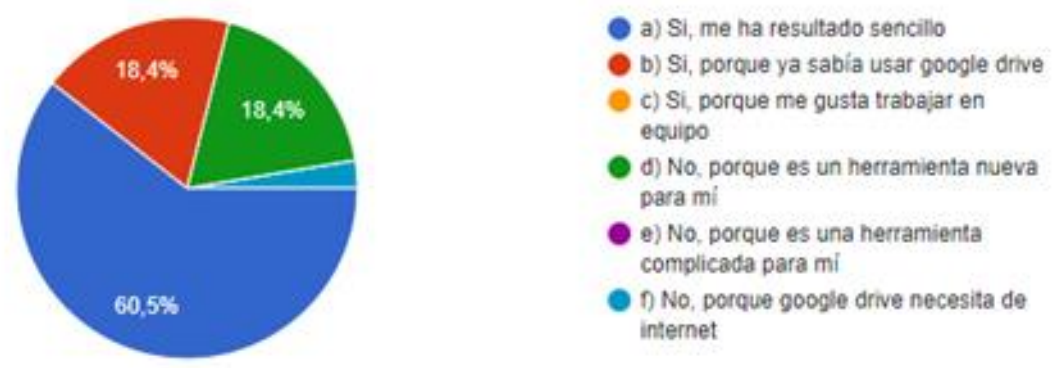

Fuente: Elaboración propia

En otro orden de ideas, $21 \%$ de los alumnos considera que mejoró la comunicación entre sus compañeros al usar Google Drive, mientras que 26.4 \% cree que poco o nada mejoró la comunicación.

Figura 10. ¿Mejoró la comunicación con tus compañeros al usar Google Drive?

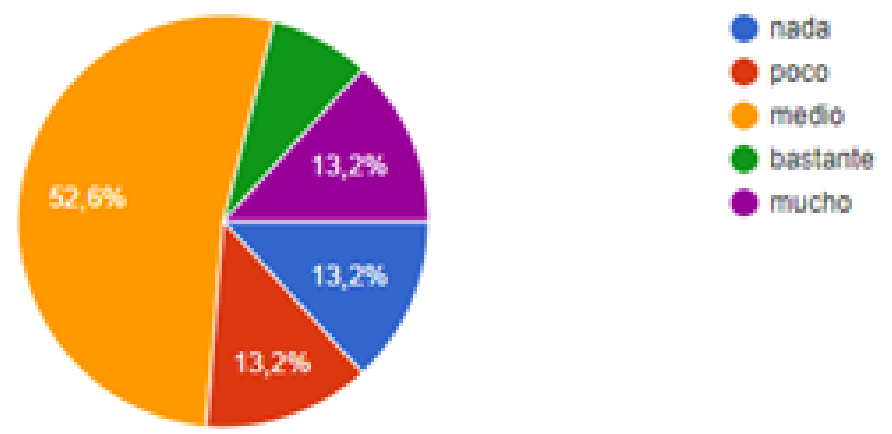

Fuente: Elaboración propia

Finalmente, $71 \%$ de los participantes expresó que la principal ventaja de Google Drive es trabajar con otras personas aunque no estén reunidos de manera presencial. 


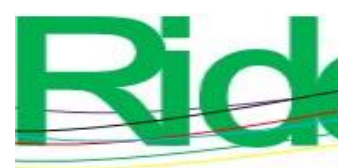

Revista Iberoamericana para la Investigación y el Desarrollo Educativo ISSN $2007-7467$

Figura 11. ¿Cuál de las siguientes ventajas de Google Drive señalarías como más importante?

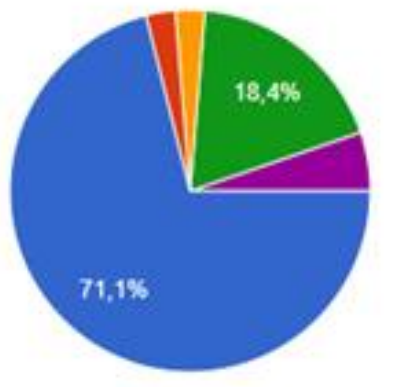

a) Trabajar con otras personas, aunque no estemos reunidos

b) Poder utilizar google drive para guardar mis archivos en la nube

c) Poder crear documentos y compartirlos en la nube

d) Poder revisar desde google drive los avances y aportaciones de cada uno $d$ mis compañeros

otra

Fuente: Elaboración propia

\section{Discusión}

El trabajo colaborativo ha encontrado en Google Drive y en otras herramientas digitales un espacio que puede potencializar sus objetivos, al facilitar la comunicación entre los miembros de grupo, pudiendo ser hasta en tiempo real; el intercambio de documentos (considerando que también se pueden archivar y acceder a ellos), así como la creación y edición de documentos donde todos los participantes pueden contribuir de manera precisa en ello, respondiendo así a una de las preguntas de investigación: ¿la creación y edición de documentos con la herramienta Google drive por equipos de alumnos universitarios permite el trabajo colaborativo?

Se destaca el tema del diseño instruccional para el logro de los objetivos planteados deberá de ser muy preciso y siguiendo una metodología probada.

Tomando en cuenta que la realización de este estudio se llevó a cabo durante el año 2019, no se puede dejar a un lado que la pandemia del COVID19 abrió un abanico de posibilidades de los recursos tecnológicos para la interacción e interactividad en los procesos de enseñanzaaprendizaje, la forma sincrónica de estos procesos, así como la implementación acelerada de dichos recursos, donde se hace pertinente el análisis de la práctica de la enseñanza, de la práctica del aprendizaje, de los resultados de estos procesos, etc., quedando así los cuestionamientos para futuras investigaciones. 


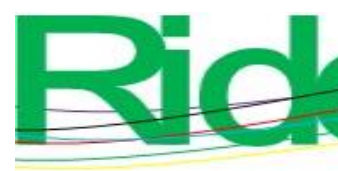

Revista Iberoamericana para la Investigación y el Desarrollo Educativo ISSN 2007-7467

\section{Conclusiones}

Google Drive resultó de gran ayuda para desarrollar cada una de las tareas indicadas. De hecho, los alumnos identificaron como atractivo su uso, pues al poder compartir y modificar documentos en la nube pudieron vencer las barreras de los horarios y de la presencia física. Esto es particularmente significativo cuando los participantes viven en comunidades apartadas de la universidad. En pocas palabras, este tipo de herramientas digitales colaborativas deben ser aprovechadas para potenciar los procesos de enseñanza y aprendizaje.

Sin embargo, también vale acotar algunas complicaciones que pueden surgir con el trabajo en equipo, entre las que sobresalen casos donde la comunicación y las aportaciones de los miembros quedaban registradas, lo que obliga a resolver los desacuerdos de manera personalizada. Aun así, la valoración de los alumnos sobre la herramienta fue excelente para trabajar de forma colaborativa, ya que se podían compartir documentos y tener una carpeta para reunir los archivos de cada uno de los integrantes.

El trabajo colaborativo, en definitiva, ha encontrado en Google Drive y en otras herramientas digitales similares un espacio para potenciar sus objetivos, pues facilita la comunicación entre los miembros de un grupo en tiempo real. De hecho, con estos recursos gratuitos se pude promover no solo un mayor intercambio de información, sino también la creación y edición de documentos, los cuales pueden contener ideas más sólidas gracias a la participación de varias personas, cada una de las cuales tendrá su propio punto de vista.

\section{Futuras líneas de investigación}

Conocer las estrategias más adecuadas con que los alumnos pueden aprender es un reto que se debe asumir con el apoyo de las nuevas tecnologías. Por eso, se podrían desarrollar más iniciativas que fomenten las sesiones virtuales sincrónicas y asincrónicas, así como la educación móvil y el acceso y distribución de contenidos en la nube, entre otros temas de interés. 


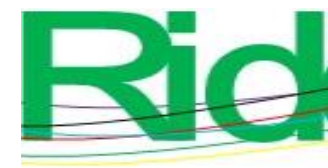

de literatura. TecnoLógicas,
Revista Iberoamericana para la
Investigación y el Desarrollo Educativo
ISSN $2007-7467$

21(41). Recuperado

de

https://revistas.itm.edu.co/index.php/tecnologicas/article/view/731

Reyero Sáez, M. R. (2019). La educación constructivista en la era digital. Revista Tecnología,

Ciencia y Educación, (12). Recuperado de https://tecnologia-cienciaeducacion.com/judima/index.php/TCE/article/view/244

Ríos, J., Chamba, L., Zumba, M. y Pardo, M. (2019). Aplicación de TICS y m-learning para mejorar el aprendizaje colaborativo e interacción mediante la plataforma Nearpod. Conferência Ibérica de Sistemas e Tecnologias de Informação. Proceedings, 1-6.

Romero, V., Romero, M., Toala F., Castro J., Pin, A., Campozano Y. y Gruezo, O. (2019). El flipped learning, el aprendizaje colaborativo y las herramientas virtuales en la educación. España: Editorial Área de Innovación y Desarrollo, S. L.

Rosetti, S., García, M. y Rojas, I. (2021). Evaluación de la implementación de un objeto de aprendizajedesarrollado contecnología h5p. Vivat Academia. RevistadeComunicación.

Rossetti López, S.-R., García Ramírez, M. T., y Rojas Rodríguez, I.-S. (2021). Evaluación de la implementación de un objeto de aprendizaje desarrollado con tecnología H5P. Vivat Academia, (154) e 1124. https://doi.org/10.15178/va.2021.154.e1224

Thomas, T. A. (2014). Developing team skills through a collaborative writing assignment. Assessment \& Evaluation in Higher Education, 39(4). 777-789. Doi: doi:10.1080/02602938.2013.850587 


\begin{tabular}{|c|c|}
\hline Rol de Contribución & Autor (es) \\
\hline Conceptualización & MIGUEL ÁNGEL RANGEL ROMERO \\
\hline Metodología & $\begin{array}{l}\text { MIGUEL ÁNGEL RANGEL ROMERO (principal) } \\
\text { ADRIANA LORENA ÍNIIGUEZ CARRILLO (apoyo) }\end{array}$ \\
\hline Software & ABRAHAM JAIR LÓPEZ VILLALVAZO \\
\hline Validación & $\begin{array}{l}\text { MIGUEL ÁNGEL RANGEL ROMERO (apoyo) } \\
\text { ADRIANA LORENA ÍNIIGUEZ CARRILLO (apoyo) }\end{array}$ \\
\hline Análisis Formal & $\begin{array}{l}\text { MIGUEL ÁNGEL RANGEL ROMERO (principal) } \\
\text { ADRIANA LORENA ÍNIIGUEZ CARRILLO (apoyo) }\end{array}$ \\
\hline Investigación & MIGUEL ÁNGEL RANGEL ROMERO \\
\hline Recursos & ABRAHAM JAIR LÓPEZ VILLALVAZO \\
\hline Curación de datos & $\begin{array}{l}\text { ABRAHAM JAIR LÓPEZ VILLALVAZO (apoyo) } \\
\text { ADRIANA LORENA ÍÑIGUEZ CARRILLO (principal) }\end{array}$ \\
\hline $\begin{array}{l}\text { Escritura - Preparación del } \\
\text { borrador original }\end{array}$ & $\begin{array}{l}\text { MIGUEL ÁNGEL RANGEL ROMERO (principal) } \\
\text { ADRIANA LORENA ÍNIIGUEZ CARRILLO (apoyo) }\end{array}$ \\
\hline $\begin{array}{l}\text { Escritura - Revisión y } \\
\text { edición }\end{array}$ & $\begin{array}{l}\text { MIGUEL ÁNGEL RANGEL ROMERO (principal) } \\
\text { ADRIANA LORENA ÍNIGUEZ CARRILLO (apoyo) } \\
\text { ABRAHAM JAIR LÓPEZ VILLALVAZO (apoyo) }\end{array}$ \\
\hline Visualización & MIGUEL ÁNGEL RANGEL ROMERO \\
\hline Supervisión & ADRIANA LORENA ÍÑIGUEZ CARRILLO \\
\hline Administración de Proyectos & MIGUEL ÁNGEL RANGEL ROMERO \\
\hline Adquisición de fondos & ABRAHAM JAIR LÓPEZ VILLALVAZO \\
\hline
\end{tabular}

